Europhys. Lett., 62 (5), pp. 636-642 (2003)

\title{
Husimi functions at dielectric interfaces: Inside-outside duality for optical systems and beyond
}

\author{
M. Hentschel ${ }^{1}\left(^{*}\right)$, H. Schomerus ${ }^{1}$ and R. Schubert ${ }^{2}$ \\ 1 Max-Planck-Institut für Physik komplexer Systeme \\ Nöthnitzer Str. 38, D-01187 Dresden, Germany \\ 2 Service de Physique Théorique, CEA/DSM/SPhT \\ Unité de recherche associée au CNRS, CEA/Saclay \\ F-91191 Gif-sur-Yvette Cédex, France
}

(received 23 December 2002; accepted in final form 4 April 2003)

PACS. 03.65.Sq - Semiclassical theories and applications.

PACS. 03.65. Nk - Scattering theory.

PACS. 42.25. $-\mathrm{p}-$ Wave optics.

\begin{abstract}
We introduce generalized Husimi functions at the interfaces of dielectric systems. Four different functions can be defined, corresponding to the incident and departing wave on both sides of the interface. These functions allow to identify mechanisms of wave confinement and escape directions in optical microresonators, and give insight into the structure of resonance wave functions. Off resonance, where systematic interference can be neglected, the Husimi functions are related by Snell's law and Fresnel's coefficients.
\end{abstract}

Optical microresonators receive growing interest over the last years, because of the intricate interplay of shape (leading to irregular classical ray dynamics), openness of the system (offering means of excitation and escape), and the wave nature of the field. This interplay, together with the promising prospect of applications in future communication devices, has stimulated experiments $[1-3]$ as well as theoretical investigations $[4,5]$ that were based on concepts well known from scattering theory, classical ray dynamics, semiclassics, and quantum chaos [6]. A particularly useful tool to study waves in dynamical systems is the Husimi representation of the wave function in classical phase space [7]. The Husimi function is obtained from the overlap of the wave with a coherent state (a minimal-uncertainty wave packet). It constitutes a quasiprobability in phase space, which acquires an intuitive quasi-classical character in the semiclassical limit of short wavelength. The Husimi representation has been widely used in the context of quantum chaos, especially in order to study semiclassical aspects of model systems that usually are closed billiards. A particularly useful modification of this approach was introduced by Crespi et al. [8], who derived a Husimi representation in a reduced phase space, namely on the Poincaré surface of section at the boundary of the system.

Here, we are interested in optical microsystems [9] where the boundary is not a hard wall but of dielectric nature, i.e., an interface of two materials with different refractive indices (such as glass and air). This implies that the system is open (light can cross the boundary), and

(*) Present address: Department of Physics, Duke University - Box 90305, Durham, NC 27708-0305, USA. 


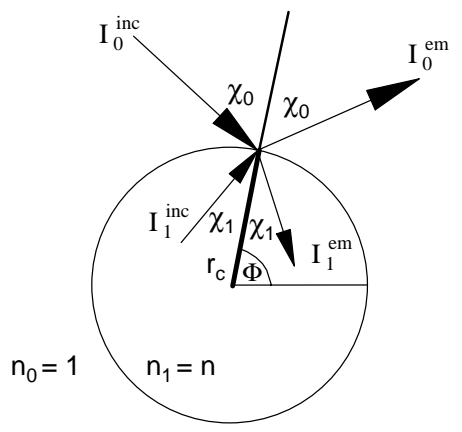

Fig. 1 - Refractive-index boundary of a dielectric system.

that the boundary conditions are neither of Dirichlet nor of von Neumann type but of a mixed type that follows from Maxwell's equations. In this situation attention is paid not only to the confinement of the (electromagnetic) waves, by a reflection mechanism which now depends of the angle of incidence, but also to the radiation characteristics of the device, which are the most interesting properties both in recent experiments [3] as well as in the design of microlasers - one wishes to identify the principal radiation directions and the locations on the boundary where the light emanates from. This calls for a Husimi representation at dielectric interfaces. Although probability densities on the boundary of open systems have been presented in, e.g., the context of microlasers [3,4], a rigorous definition of Husimi functions which accounts for the mixed boundary conditions was missing so far. As a matter of fact, the system was treated as a closed system, and conclusions have been drawn by adding the laws of reflection and refraction by hand. Moreover, the previous approaches suffer from the fact that the incident and emerging wave components cannot be discriminated by the conventional Husimi representation, a deficiency that becomes even more troublesome when the wavelength is comparable to the system size and ray optics does not apply. Besides the conceptual shortcoming, this complicated the determination of the radiation directions of optical microresonators.

In this paper we introduce four Husimi representations $H_{j}^{\text {inc,em }}$ of the wave function, which are appropriate quasiprobabilities at dielectric interfaces. They correspond to the intensity $I_{j}^{\mathrm{inc}, \mathrm{em}}=H_{j}^{\mathrm{inc}, \mathrm{em}} \mathrm{d} \phi \mathrm{d} \sin \chi_{j}$ of incident (inc) and emerging (em) rays inside $(j=1)$ and outside $(j=0)$ the interface, where the rays are parameterized by the angles $\phi$ and $\chi_{j}$ as illustrated in fig. 1. In the regime of ray optics it will be demonstrated that these Husimi functions are related across the interface via Fresnel's formulas for the intensities, with phase space being deformed according to Snell's law. This connection can be seen as a new variant of the inside-outside duality [10]. However, ray optics only applies when the wavelength is short and when systematic interference effects can be neglected. The Husimi functions do not require these limitations and develop their full predictive power especially when systematic interference effects lead to strong deviations from Fresnel's and Snell's laws. In particular, ray optics breaks down close to resonances, where the internal part of the scattering wave function is known to be almost independent of the incoming wave that excites the system. The Husimi functions still provide an accurate representation of the wave function (in particular, they nicely display the radiation directions of the field). We illustrate these features using the dielectric circular disk and an annular system as examples [11].

Microresonators and ray optics. - Consider the light that illuminates and permeates a dielectric system confined by a refractive-index boundary, as shown in fig. 1 . The disk and the annulus both are confined by a circular dielectric interface of radius $r_{\mathrm{c}} \equiv 1$. Polar coordinates 
$r, \Phi$ will be used to parameterize position space. We distinguish four wave components: The incident (inc) wave and the emerging (em) wave on both sides of the interface (region 0 with refractive index $n_{0}=1$ outside the microresonator, region 1 with $n_{1} \equiv n$ inside the microresonator). In the regime of ray optics, the wave is represented by rays, where the angles of incident and the emerging rays are related by the laws of reflection and Snell's law, $n \sin \chi_{1}=\sin \chi_{0}$. For our circular interfaces Snell's law is equivalent to conservation of the angular-momentum variable $m=k_{j} \sin \chi_{j}$, where $k_{0}$ and $k_{1}=n k_{0}$ are the wave number in each region. The ray intensities on either side are connected by

$$
\begin{aligned}
& I_{0}^{\mathrm{em}}=R_{0}\left(\chi_{0}\right) I_{0}^{\mathrm{inc}}+T_{1}\left(\chi_{1}\right) I_{1}^{\mathrm{inc}}, \\
& I_{1}^{\mathrm{em}}=R_{1}\left(\chi_{1}\right) I_{1}^{\mathrm{inc}}+T_{0}\left(\chi_{0}\right) I_{0}^{\mathrm{inc}},
\end{aligned}
$$

where the reflection and transmission coefficients $R_{j}$ and $T_{j}$ are related by

$$
R_{0}\left(\chi_{0}\right)=R_{1}\left(\chi_{1}\right) \equiv R, \quad T_{0}\left(\chi_{0}\right)=T_{1}\left(\chi_{1}\right)=1-R,
$$

with $R=\sin ^{2}\left(\chi_{1}-\chi_{0}\right) / \sin ^{2}\left(\chi_{1}+\chi_{0}\right)$ according to Fresnel's law [11,12].

Husimi functions at a dielectric interface. - Husimi functions are originally defined as the overlap of a coherent state (a minimal-uncertainty wave packet) $\xi$ with an eigenstate $\Psi$ of the system [7]. They are interpreted as probability densities (with quantum-mechanical origin) in the full classical phase space (at the coordinates $r, \Phi$, momentum components $k \sin \chi, k \cos \chi$ at which the coherent state is centered). The Husimi function at the system boundary of closed systems with either von Neumann or Dirichlet boundary conditions was introduced in refs. $[8,13]$ by projection of the conventional Husimi function from full phase space onto the reduced phase space at the boundary $r=r_{\mathrm{c}}$ with coordinates $\phi=\Phi$ and $\sin \chi_{j}$ [14]. As we will show now, the four different Husimi functions (corresponding to the incident and emerging wave at both sides of a dielectric interface) in the reduced phase space at the boundary of an open system can be constructed rigorously by the same projection procedure when the appropriate boundary conditions are employed [13].

Both in the interior region $(j=1)$ and the exterior region $(j=0)$, the wave function $\Psi_{j}(r, \Phi)$ satisfies the Helmholtz equation $\left[\Delta+k_{j}^{2}\right] \Psi_{j}(r, \Phi)=0$. Hence we can use Green's formula to express $\Psi_{j}$ as an integral over the boundary, involving both $\Psi_{j}$ and its normal (radial) derivative $\Psi_{j}^{\prime}$. For example, in the interior this results in

$$
\begin{aligned}
\Psi_{1}(r, \Phi)=\int_{0}^{2 \pi} \mathrm{d} \phi r_{\mathrm{c}}[ & G_{1}^{\mathrm{inc}}\left(\boldsymbol{x}(r, \Phi)-\boldsymbol{x}\left(r_{\mathrm{c}}, \phi\right)\right) \Psi_{1}^{\prime}\left(\boldsymbol{x}\left(r_{\mathrm{c}}, \phi\right)\right)- \\
& \left.-\partial_{r} G_{1}^{\mathrm{inc}}\left(\boldsymbol{x}(r, \Phi)-\boldsymbol{x}\left(r_{\mathrm{c}}, \phi\right)\right) \Psi_{1}\left(\boldsymbol{x}\left(r_{\mathrm{c}}, \phi\right)\right)\right],
\end{aligned}
$$

where we encounter the position representation (in Cartesian coordinates $\boldsymbol{x}$ ) of the retarded Green's function

$$
G_{j}^{\mathrm{inc}}=\lim _{\varepsilon \rightarrow 0^{+}} \frac{1}{\Delta+k_{j}^{2}+i \varepsilon} .
$$

Similarly, one can use the advanced Green's function $G_{j}^{\mathrm{em}}$, and having both options at our disposal indeed allows us to distinguish between incident and emerging waves. The squared overlap $\left|\left\langle\Psi_{j}, \xi_{(\boldsymbol{p}, \boldsymbol{q})}\right\rangle\right|^{2}$ of $\Psi_{j}$ with a coherent state $\xi_{(\boldsymbol{p}, \boldsymbol{q})}((\boldsymbol{p}, \boldsymbol{q})$ denote the momentum and position around which the state is localized) can be transformed into the Husimi quasiprobability

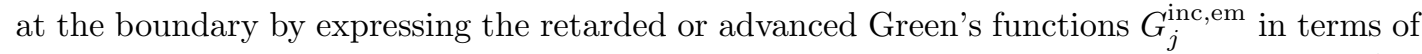
the half-sided temporal Fourier transformations of the time evolution operator $\boldsymbol{U}(t)=e^{\mathrm{i} \Delta t / k_{0}}$, 
$G_{j}^{\text {inc,em }}=\mp \mathrm{i} / k_{0} \int_{0}^{\infty} e^{ \pm \mathrm{i} k_{0} n_{j}^{2} t} \boldsymbol{U}( \pm t) \mathrm{d} t$. Indeed, the time evolution operator acts as a unitary transformation that propagates the coherent state to and across the boundary, still preserving its coherent nature. In practical terms, we now have to calculate the overlap with a Gaussian beam emanating from the spatial center of the minimal-uncertainty wave packet $\xi_{(\boldsymbol{p}, \boldsymbol{q})}$, with the corresponding momentum backward in time. This evaluation can be performed by a semiclassical (saddle point) approximation (the details will be given in [13]), and leads to the relation

$$
\left(\frac{k_{0}}{2 \pi}\right)^{2}\left|\left\langle\Psi_{j}, \xi_{( \pm \boldsymbol{p}, \boldsymbol{q})}\right\rangle\right|^{2} \approx \frac{k_{0}^{1 / 2}}{4 \pi^{1 / 2}} e^{-k_{0}\left(n_{j}-|\boldsymbol{p}|\right)^{2}} H_{j}^{\mathrm{inc}(\mathrm{em})}\left(\phi, \sin \chi_{j}\right) .
$$

Here $\boldsymbol{p}$ is the momentum vector of the incoming ray which meets the boundary at the point $\phi$ with angle $\chi_{j}$ relative to the normal, $\boldsymbol{q}$ is any position on this incoming ray, and the four different Husimi functions on the interface are given by

$$
H_{j}^{\mathrm{inc}(\mathrm{em})}\left(\phi, \sin \chi_{j}\right)=\frac{k_{j}}{2 \pi}\left|(-1)^{j} \mathcal{F}_{j} h_{j}\left(\phi, \sin \chi_{j}\right)+(-) \frac{\mathrm{i}}{k_{0} \mathcal{F}_{j}} h_{j}^{\prime}\left(\phi, \sin \chi_{j}\right)\right|^{2},
$$

with the angular-momentum-dependent weighting factor $\mathcal{F}_{j}=\sqrt{n_{j} \cos \chi_{j}}[15]$. The functions

$$
\begin{aligned}
& h_{j}\left(\phi, \sin \chi_{j}\right)=\int_{0}^{2 \pi} \mathrm{d} \Phi r_{\mathrm{c}} \Psi_{j}\left(r_{\mathrm{c}}, \Phi\right) \xi\left(\Phi ; \phi, \sin \chi_{j}\right), \\
& h_{j}^{\prime}\left(\phi, \sin \chi_{j}\right)=\int_{0}^{2 \pi} \mathrm{d} \Phi r_{\mathrm{c}} \Psi_{j}^{\prime}\left(r_{\mathrm{c}}, \Phi\right) \xi\left(\Phi ; \phi, \sin \chi_{j}\right)
\end{aligned}
$$

are the overlaps of the wave function $\Psi$ and its normal (radial) derivative $\Psi^{\prime}$, taken on the respective side $j$ of the interface, with the minimal-uncertainty wave packet

$$
\xi\left(\Phi ; \phi, \sin \chi_{j}\right)=(\sigma \pi)^{-\frac{1}{4}} \sum_{l} e^{-\frac{1}{2 \sigma}(\Phi+2 \pi l-\phi)^{2}-\mathrm{i} k_{0} \sin \chi_{j}(\Phi+2 \pi l)}
$$

(a periodic function in $\Phi$ ), which is centered around $\left(\phi, \sin \chi_{j}\right)$. The parameter $\sigma$ controls its extension in the $\phi$-direction, thereby also fixing the uncertainty in $\sin \chi_{j}$. We set $\sigma=\sqrt{2} / k_{1}$. The scaling with $k_{1}$ results in the same resolution in $\phi$ for all four Husimi functions.

The relation (6) provides a direct physical interpretation of the four Husimi functions (7) on the interface as intensities of the incoming and outgoing radiation field from $\phi$ in the directions $\chi_{j}$, and this is our motivation for choosing them as the proper Husimi functions on the interface.

Inside-outside duality. - As a consequence of Maxwell's equations we find the identities $h_{0}\left(\phi, \sin \chi_{0}\right)=h_{1}\left(\phi, \sin \chi_{1}\right), h_{0}^{\prime}\left(\phi, \sin \chi_{0}\right)=h_{1}^{\prime}\left(\phi, \sin \chi_{1}\right)$, where the angles $\chi_{j}$ are related by Snell's law. From these relations it follows that our Husimi functions strictly fulfill the condition of intensity conservation,

$$
n H_{0}^{\mathrm{em}}\left(\phi, \sin \chi_{0}\right)+H_{1}^{\mathrm{em}}\left(\phi, \sin \chi_{1}\right)=n H_{0}^{\mathrm{inc}}\left(\phi, \sin \chi_{0}\right)+H_{1}^{\mathrm{inc}}\left(\phi, \sin \chi_{1}\right),
$$

where the factor $n=\mathrm{d} \sin \chi_{0} / \mathrm{d} \sin \chi_{1}$ accounts for the phase-space deformation by Snell's law [16]. Additional relations between the Husimi functions can be anticipated in the regime of ray optics: The intensities on one side of the interface should be related to the intensities on the other side by eqs. (1), (2). We then expect validity of the resulting inside-outside duality relations

$$
\begin{aligned}
& H_{0}^{\mathrm{em}} \approx S\left(H_{0}^{\mathrm{em}}\right)=\frac{1}{n} \frac{1-2 R}{1-R} H_{1}^{\mathrm{inc}}+\frac{1}{n} \frac{R}{1-R} H_{1}^{\mathrm{em}}, \\
& H_{0}^{\mathrm{inc}} \approx S\left(H_{0}^{\mathrm{inc}}\right)=-\frac{1}{n} \frac{R}{1-R} H_{1}^{\mathrm{inc}}+\frac{1}{n} \frac{1}{1-R} H_{1}^{\mathrm{em}},
\end{aligned}
$$



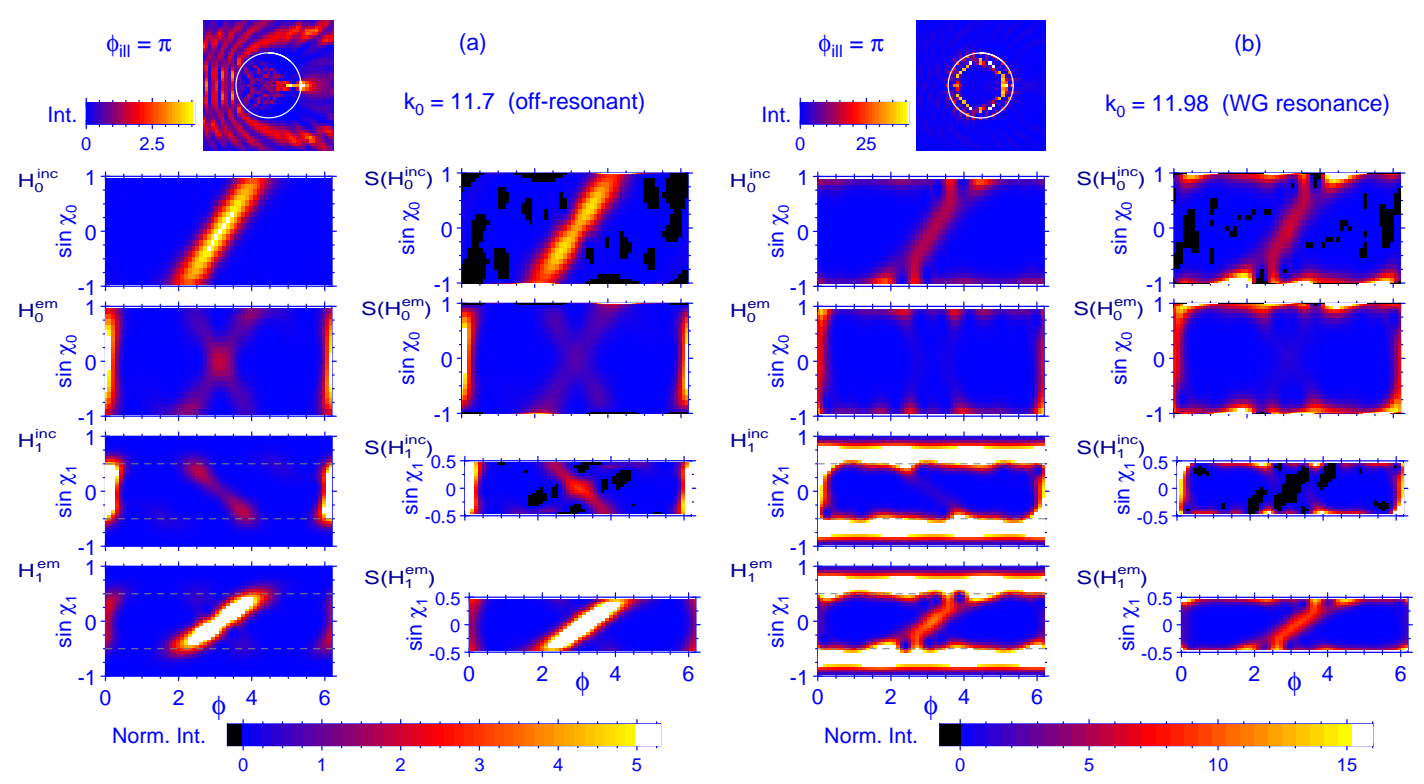

Fig. 2 - (a) Exact (left) and reconstructed (right) Husimi functions for the circular dielectric disk $(n=2)$ illuminated by a plane wave at an off-resonant frequency. The top panel shows the scattering wave function in real space. The exact Husimi functions are obtained from eq. (7). The reconstructed Husimi functions are obtained by eqs. (12)-(15). Negative Husimi densities are shown in black. The dashed lines in the panels for region 1 mark the critical angle of incidence for total internal reflection. (b) Same as (a), but for illumination at a resonance frequency.

which express the Husimi functions in region 0 by the Husimi functions in region 1 . The notation $S(\cdot)$ indicates that the approximation is of semiclassical (short wavelength) nature; most notably, the Husimi functions (intensities) are added incoherently. The duality relations can also be inverted,

$$
\begin{aligned}
& H_{1}^{\mathrm{em}} \approx S\left(H_{1}^{\mathrm{em}}\right)=n \frac{1-2 R}{1-R} H_{0}^{\mathrm{inc}}+n \frac{R}{1-R} H_{0}^{\mathrm{em}}, \\
& H_{1}^{\mathrm{inc}} \approx S\left(H_{1}^{\mathrm{inc}}\right)=-n \frac{R}{1-R} H_{0}^{\mathrm{inc}}+n \frac{1}{1-R} H_{0}^{\mathrm{em}} .
\end{aligned}
$$

However, the Husimi functions in region 1 can only be reconstructed in the strip $\left|\sin \chi_{1}\right|<1 / n$, because the rest of phase space is isolated from region 0 by total internal reflection.

The duality relations are exactly fulfilled in two simple cases, namely, if one incident or emerging wave vanishes or when the two incident waves have the same intensity (the two emerging waves then have the same intensity, as well). We now test the duality relations in more general situations.

The panels on the left in fig. 2(a) show the exact Husimi functions from eq. (7) for the case of the circular disk which is excited by a plane wave at an off-resonant excitation frequency. The incident plane wave is clearly visible in the Husimi function $H_{0}^{\text {inc }}$, around the polar angle $\phi=\phi_{\text {ill }}=\pi$, while the focal point of the dielectric disk results in a bright spot in $H_{0}^{\mathrm{em}}$ that is located around $\phi=0$. There is a close correspondence between the Husimi functions of the incident and emerging waves, and the deformation of phase space by the stretching factor $n$ of Snell's law is clearly visible.

The right panels of fig. 2(a) show for comparison the predictions of eqs. (12)-(15). In the 

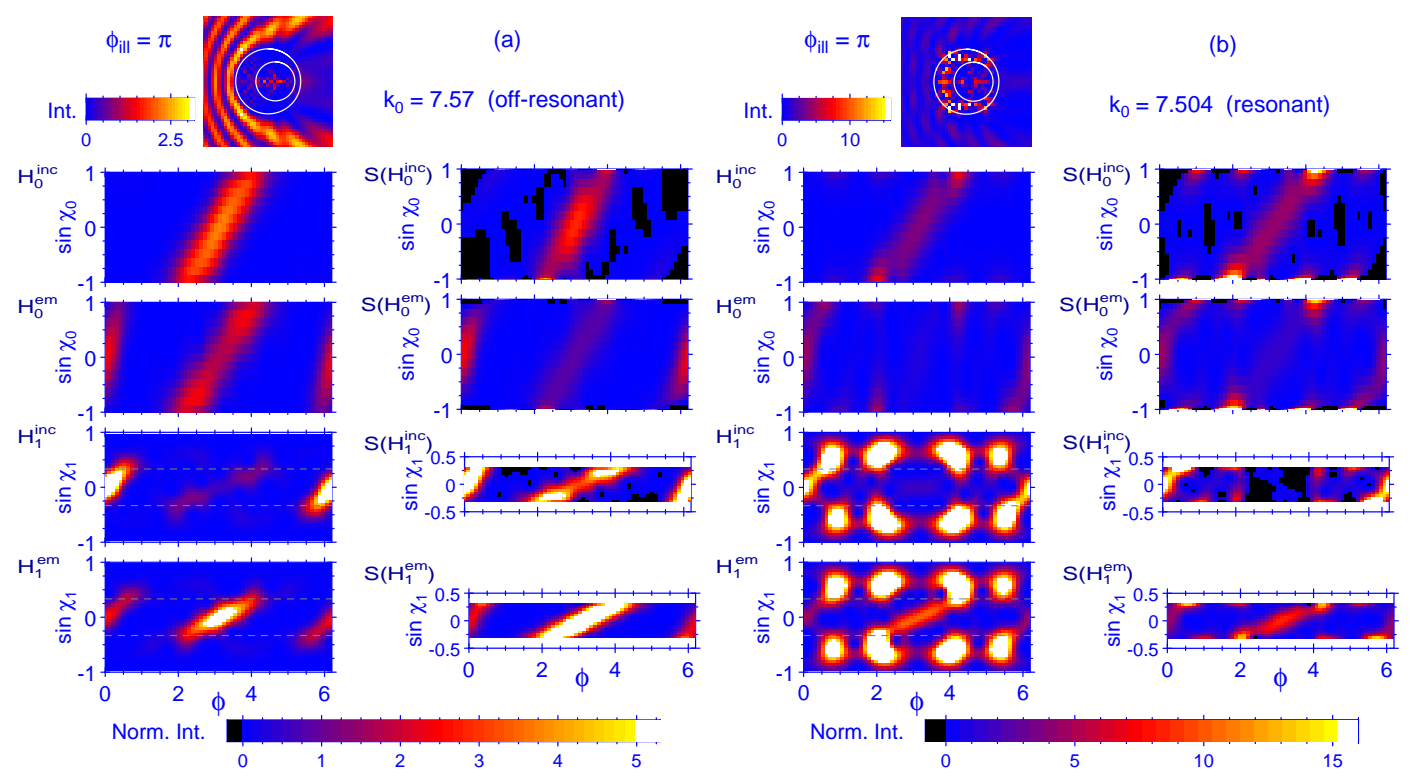

Fig. 3 - Same as fig. 2, but for a dielectric annulus (refractive indices $n_{0}=1$ outside, $n_{1}=3$ in the annulus, $n_{2}=6$ in the inner disk, radii $R_{1}=1, R_{2}=0.6$, displaced by $\left.\delta=0.22\right)$.

reconstruction we used the slightly modified semiclassical versions of Fresnel's coefficients and Snell's laws given in ref. [12], which are appropriate for the present case of a curved interface. We observe a good qualitative and quantitative agreement with the exact Husimi functions. Regions with unphysical negative intensities are small. The most interesting deviations between the exact and the reconstructed Husimi functions occur around the central spot at $\phi=\pi, \sin \chi_{0}=0$, where the incoherent prediction of eqs. (12) and (13) underestimates the exact Husimi densities $H_{0}^{\text {inc }}, H_{0}^{\mathrm{em}}$. It is complemented by overestimated intensities $H_{1}^{\mathrm{inc}}, H_{1}^{\mathrm{em}}$ in the same area of phase space. These deviations arise from a Fabry-Pérot-like systematic interference which is constructive in backward direction at the presently chosen frequency. At other frequencies the interference is destructive, and both cases alternate periodically.

Resonances. - Figure 2(b) displays the situation for excitation at a frequency which is close to a narrow resonance, a whispering gallery (WG) mode located around $\sin \chi_{1}=0.667$. The top panel shows that the wave function is now well confined inside the disk (region 1). Correspondingly, the Husimi functions $H_{1}^{\text {inc }}$ and $H_{1}^{\mathrm{em}}$ noticeably exceed the Husimi functions $H_{0}^{\text {inc }}$ and $H_{0}^{\mathrm{em}}$. Moreover, the Husimi functions $H_{1}^{\text {inc }}$ and $H_{1}^{\mathrm{em}}$ are dominated by the characteristics of the resonance wave function and consequentially are almost independent of the choice of the exciting wave. (The remnants of the exciting plane can be identified when comparing fig. 2(b) with (a).) Hence the reconstructed Husimi functions deviate noticeably from the exact Husimi functions around $\left|\sin \chi_{1}\right| \sim 1 / n$. This is no surprise, since resonances are formed by systematic constructive interference, and incoherent ray optics cannot be expected to apply under these circumstances. Most importantly, by principle, the confined wave intensity in the region $\left|\sin \chi_{1}\right|>1 / n$ cannot be reconstructed because classically [4] no refracted rays ever reach this region (which is dark off resonance). On the other hand, the exact Husimi functions display nicely all the features of the resonance wave function in phase space.

Finally, let us illustrate the usefulness of the Husimi functions (7) also for a more complex system than the circular disk, the annular system formed by regions of different refractive 
indices that are confined by two eccentric disks. The ray optics in this system corresponds to nonintegrable dynamics in phase space, which allows for a multifaceted set of resonance wave functions [5]. Off resonance (fig. 3(a)) the scattering wave function enters the dielectric system only barely, and the situation is similar to the circular disk because the internal disk is not explored extensively. At resonance the situation is very different. Figure 3(b) shows a typical resonance wave function in real space and its Husimi representation in phase space. The intensity of the resonance wave function is concentrated on straight segments which can be identified as a short stable periodic trajectory of the corresponding classical ray dynamics. The Husimi functions display a strong intensity exactly in the vicinity of this trajectory in classical phase space.

In conclusion, we introduced four Husimi representations of the scattering wave function at the interfaces of dielectric microresonators, corresponding to the incident and emerging waves at both sides of the interface. These Husimi functions are easily computed from the wave function and have many desirable properties: They are related by the laws of Fresnel and Snell in the ray-optics regime (i.e., short and off-resonant wavelength) and generally provide valuable detailed insight into the wave dynamics in complex dielectric systems, most notably even close to resonances where ray optics breaks down.

$$
* * *
$$

We gratefully acknowledge helpful discussions with J. Wiersig, A. BÄCKER, C. MiniATURA and D. UlLmo.

\section{REFERENCES}

[1] Doya V., Legrand O., Mortessagne F. and Miniatura C., Phys. Rev. Lett., 88 (2002) 014102.

[2] Lee S.-B. et al., Phys. Rev. Lett., 88 (2002) 033903.

[3] Rex N. B. et al., Phys. Rev. Lett., 88 (2002) 094102.

[4] Nöckel J. U. and Stone A. D., Nature, 385 (1997) 45.

[5] Hentschel M. and Richter K., Phys. Rev. E, 66 (2002) 056207.

[6] Stöckmann H.-J., Quantum Chaos (Cambridge University Press, Cambridge) 1999.

[7] Leboeuf P. and Saraceno M., J. Phys. A, 23 (1990) 1745; Husimi K., Proc. Phys. Math. Soc. Jpn., 22 (1940) 246.

[8] Crespi B., Perez G. and Chang S.-J., Phys. Rev. E, 47 (1993) 986.

[9] In two dimensions, optical and quantum-mechanical systems (described by the Helmholtz and the Schrödinger equation, respectively) are equivalent, see [6].

[10] Doron E. and Smilansky U., Nonlinearity, 5 (1992) 1055; Hornberger K. and Smilansky U., Phys. Rev. Lett., 88 (2002) 024101.

[11] For simplicity, we only consider the case that the electric field is polarized perpendicular to the resonator plane.

[12] Hentschel M. and Schomerus H., Phys. Rev. E, 65 (2002) 045603(R).

[13] SCHUberT R., in preparation.

[14] All considerations are easily extended to non-spherical interfaces when one replaces the polar angle $\phi$ by the arc length $s$ along the interface.

[15] The unphysical singularity $\mathcal{F}^{-1} \rightarrow \infty$ for $\chi \rightarrow \pi / 2$ is regularized by the corrections to the semiclassical derivation of eq. (7). In our calculations we regularized $\mathcal{F}$ by setting a lower cutoff value $\mathcal{F}_{\mid}>\left[4 n_{j}^{2} /\left(k_{j} r_{\mathrm{c}}\right)\right]^{1 / 4}$, which is motivated by these corrections.

[16] This equation takes another illustrative form using the local phase space measure $\mathrm{d} \phi \mathrm{d} \sin \chi_{j}$ (and dropping the arguments): $H_{0}^{\text {em }} \mathrm{d} \phi \mathrm{d} \sin \chi_{0}+H_{1}^{\text {em }} \mathrm{d} \phi \mathrm{d} \sin \chi_{1}=H_{0}^{\text {inc }} \mathrm{d} \phi \mathrm{d} \sin \chi_{0}+H_{1}^{\text {inc }} \mathrm{d} \phi \mathrm{d} \sin \chi_{1}$. 